\title{
EDUMÓVIL: INCORPORANDO LA TECNOLOGÍA MÓVIL EN LA EDUCACIÓN PRIMARIA
}

\section{(EDUMÓVIL: INCORPORATING THE MOBILE TECHNOLOGY IN PRIMARY SCHOOLS)}

\author{
Gabriel Gerónimo-Castillo \\ Everth. H. Rocha-Trejo \\ Universidad Tecnológica de la Mixteca (México)
}

\section{RESUMEN}

Este artículo versa sobre el desarrollo del proyecto Edumóvil que tiene como objetivo mejorar el proceso enseñanza-aprendizaje de los niños de nivel primaria a través de la incorporación de tecnología móvil en el aula. Edumóvil pretende explotar los beneficios que trae consigo la tecnología móvil en la educación, como el bajo costo, la movilidad, las responsabilidades individuales y la oportunidad de trabajo colaborativo. Se habla de las aplicaciones concluidas y las que se encuentran en desarrollo.

Palabras clave: educación, niños, tecnología móvil, usabilidad.

\begin{abstract}
This paper presents the Project Edumóvil, which has the objective to improve the teaching and learning processes in Mexican primary schools by integrating mobile technology into the classroom. Edumóvil aims to investigate the benefits and assets that can be obtained by using mobile technology in education: low costs, mobility, individual responsabilities and the opportunity to work in a collaborative manner. Both finalized applications as well as applications that are developed will be presented.
\end{abstract}

Key words: education, children, mobile technology, usability.

Actualmente los dispositivos móviles gozan de un gran auge en los diferentes ámbitos de la sociedad. Cada vez es mayor el número de aplicaciones que se pueden encontrar en el mercado, tanto en forma comercial como en forma gratuita. Aunado a 
esto, los niños en México y el resto del mundo se acercan cada vez más a la utilización de tecnología de punta. Por otro lado, se ha despertado gran interés por diseñar e implementar herramientas que apoyen el proceso enseñanza-aprendizaje en niños de nivel primaria, no sólo por parte de la Secretaría de Educación Pública (SEP) en México sino también por maestros, investigadores y estudiantes de nuestro país. Hasta el momento existe muy poco material en México que propicie el desarrollo del niño dentro o fuera del aula, que fomente el trabajo en forma individual y en equipo, además del aprendizaje colaborativo. Varios países como Estados Unidos, Canadá, Inglaterra y Chile han centrado su atención en fomentar el proceso enseñanzaaprendizaje colaborativo, y cuentan con investigaciones que muestran grandes beneficios de esta forma de enseñanza (Zurita, 2005, 2004; Druin, 2002). El proyecto Edumóvil busca incorporar a nuestro país en el proceso de enseñanza-aprendizaje en forma individual como en forma colaborativa, desarrollando aplicaciones móviles enfocadas a estudiantes de nivel primaria.

\section{LA ENSEÑANZA EN LA EDUCACIÓN PRIMARIA}

A nivel primaria en México la SEP dicta que el tiempo a la semana dedicado a las materias se divida en 45\% para Español, 30\% para Matemáticas y 25\% para Conocimiento del Medio. El objetivo en Español es desarrollar las capacidades de comunicación de los niños en los distintos usos de la lengua hablada y escrita. En Matemáticas deben razonar y comprender los textos para resolver problemas, y en Conocimiento del Medio el niño aprende a vincular su conocimiento con el mundo que lo rodea. Cabe una interrogante, ¿qué hace el profesor cuando se dificulta el aprendizaje a los niños? Los profesores mencionan que utilizan los métodos inductivo y constructivista, apoyándose en material concreto y objetivo, así como en juegos y actividades grupales. Los medios (pizarrón, libros, laminas, recortes, objetos, etc.) son los potenciadores cognitivos que brindan a los niños distintas estrategias y operaciones cognitivas. Existen diversas metodologías y prácticas educativas que utilizan distintos medios para transmitir conocimiento que permiten al niño aclarar y enriquecer sus ideas, una de ellas es la tecnología. El uso de la tecnología en la enseñanza genera situaciones o conceptos novedosos para que los niños manifiesten sus actitudes y sus sentimientos, además fortalece el aprendizaje colaborativo, motivando el descubrimiento de un nuevo conocimiento de lo que se quiere aprender.

Edumóvil se enfoca al trabajo en los ejes en los cuales el niño presenta dificultad de aprendizaje, brindando para esto juegos donde los niños pueden trabajar en forma individual, y otros en forma grupal, utilizando para esto el protocolo Bluetooth, y 
tomando como una buena alternativa la restricción de la tecnología donde el número de integrantes debe ser menor de 8, permitiendo así que todos puedan participar, generando talleres de enseñanza. Otorga además algunas ventajas en el proceso de aprendizaje, tales como: estímulo en la comunicación interpersonal, facilidad en el trabajo colaborativo, y seguimiento en el proceso de grupo (Calzadilla, 2002).

\section{PROPUESTA DE EDUMÓVIL}

El objetivo que persigue el proyecto Edumóvil es el de mejorar la enseñanzaaprendizaje a través de la incorporación de tecnología móvil en el aula. Entre los dispositivos contemplados se encuentran los PDAs (Personal Digital Asistants) y los teléfonos celulares. Para la comunicación de los diferentes dispositivos que permitan llevar a cabo la enseñanza-aprendizaje colaborativo dentro del aula se tiene contemplado el uso del protocolo Bluetooth, dado su bajo consumo de energía, y su alcance. Actualmente se tienen aplicaciones para PDAs, y se ha empezado a realizar algunos proyectos en teléfonos celulares utilizando la tecnología Bluetooth.

\section{ELECCIÓN DEL DISPOSITIVO}

Antes de iniciar el desarrollo de las aplicaciones se llevó a cabo pruebas de usabilidad de algunos PDAs con niños de primer grado de primaria, cuyas edades se encontraban entre los seis y siete años. Los niños en este rango de edad son los usuarios potenciales con menor edad y desarrollo psicomotriz. Las pruebas realizadas se llevaron a cabo en el Laboratorio de Usabilidad de la Universidad Tecnológica de la Mixteca a finales del 2004. Se pudo observar que por parte de las niñas el uso de botones presenta cierta dificultad, en contraste, los niños presentan una mayor habilidad en el uso de estos. Otras observaciones se presentaron en el uso del stylus (lápiz), los niños lo utilizan de manera más brusca al señalar o elegir los elementos de la pantalla, mientras que las niñas son usuarios más cuidadosos para su uso. El uso del dispositivo es mucho más sencillo para los niños que han tenido contacto con juegos de video, dado que se observó que es mucho más ágil un niño en su manera de tomar el dispositivo. Otro punto que se observó, es el peso del dispositivo, si el dispositivo es de mayor peso, el niño necesita ayuda para sostenerlo, o busca una forma de adaptarse a su uso. Esta observación fue la principal causa por la inclinación del uso de Palms como los dispositivos a utilizar en Edumóvil. Al finalizar las pruebas se puede concluir que los niños entre seis y siete años son usuarios factibles para el uso de los PDAs, y además siempre están dispuestos a explorar y ayudar (Gerónimo, 2005). 


\section{APLICACIONES EN DESARROLLO}

La SEP clasifica las asignaturas en dos bloques, el primero para alumnos de primero y segundo grado formado por las materias de Español, Matemáticas, Conocimiento del medio, Educación artística y Educación física, y el segundo bloque, para alumnos de tercero a sexto grado, con las misma materias, excepto Conocimiento del medio, la cual es dividida en las materias de Ciencias Naturales, Historia, Geografía y Educación Cívica (SEP, 2003). Hasta el momento dentro del sistema Edumóvil se han concluido aplicaciones para Matemáticas de primer año, y para Ciencias Naturales (la cual puede ser utilizada desde tercer grado a quinto grado), y se encuentran en etapa de desarrollo aplicaciones de Español, Matemáticas de segundo y tercer grado, e Historia.

\section{Matemáticas primer grado}

Los programas actuales de los diferentes grados de la educación básica en México dividen la enseñanza de las matemáticas en seis ejes: 1) los números, sus relaciones y sus operaciones, 2) medición, 3) geometría, 4) procesos de cambio, 5) tratamiento de la información y, 6) predicción y azar. Lo primero que se realiza en Edumóvil es la detección del eje donde los niños presentan mayor dificultad de aprendizaje. La identificación de este eje, se lleva a cabo por medio de encuestas realizadas en las escuelas primarias del municipio de Huajuapan de León, Oaxaca, México, cuya población de escuelas primarias es de 27, de las cuales se encuestó a 24 de ellas y a un promedio de 27 profesores. Para el primer grado el eje que se detectó con mayor dificultad de aprendizaje fue tratamiento de la información (Figura 1). En este eje se proponen actividades en las cuales se desarrolla en los niños la capacidad para resolver problemas y tratar con la información. Para lograr esto se plantea que los niños analicen y seleccionen información planteada a través de textos, imágenes u otros medios. Así como representar e identificar información a través de gráficas y tablas.

Después de detectar dicho eje, la estrategia planteada en Edumóvil para mejorar la enseñanza-aprendizaje es el desarrollo de un juego en Palms para permitir de forma fácil y entretenida el aprendizaje de dicho eje, por ejemplo, el juego Observa y Aprende que cubre aspecto del eje tratamiento de la información. 


\section{Dificultad de aprendizaje}

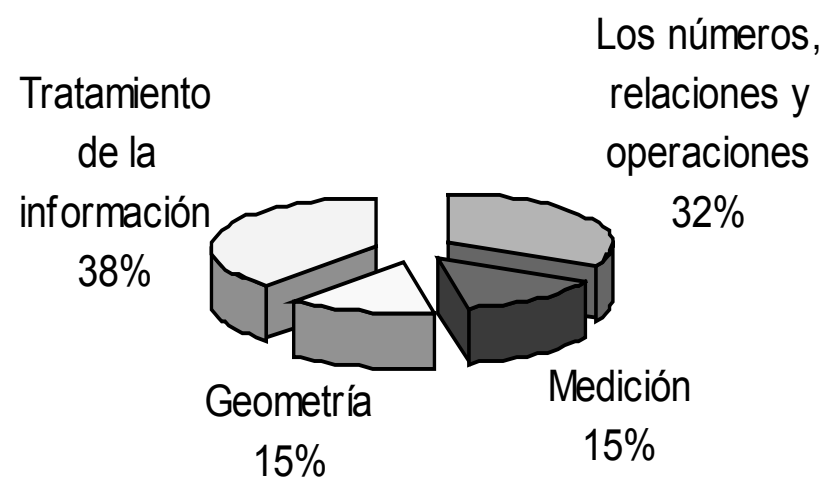

Fig. 1. Dificultad de aprendizaje (matemáticas primer grado).

Las pruebas del juego Observa y Aprende se llevaron a cabo en el laboratorio de Usabilidad de nuestra Universidad, y en la Escuela Primaria General Lázaro Cárdenas. Se notó en las pruebas que el niño se adapta rápidamente al dispositivo, existe facilidad de uso de la aplicación, y sobre todo se divierten y aprenden actividades relacionadas con el eje detectado como problemático.

\section{Español}

El objetivo central de los programas de Español de la SEP es desarrollar las capacidades de comunicación de los niños en los distintos usos de la lengua hablada y escrita. Para conseguir este objetivo, una de las metas es adquirir el hábito de la lectura. El sistema Edumóvil propone la creación de una biblioteca de lecturas animadas para PDAs, y el desarrollo de un visualizador de lecturas en los PDAs con características que los niños puedan entender y manejar de forma fácil y sencilla. Para su construcción lo primero que se realizó fue el estudio de usabilidad del dispositivo como medio de lectura, y aunado a ello la evaluación de un visualizador para observar el comportamiento del niño. Los niños a los cuales se les aplicaron las pruebas del uso de un visualizador de texto (Acrobat Reader versión 1.1.) en una 
PDA (Palm modelo Zire 71) estaban en el rango de edad de 7 a 9 años. La elección del dispositivo fue basada en los resultados obtenidos en las pruebas de usabilidad de PDAs con niños (Gerónimo, 2005). Tomando como base las habilidades detectadas y las opiniones de los niños, se encuentra en etapa de diseño el visualizador de lecturas animadas. Para realizar la planeación y ejecución de las pruebas de usabilidad se utilizaron las recomendaciones dadas por Hanna (1997). De las pruebas realizadas se notó que el tamaño de letra, en nuestro caso de 14 puntos, es pequeña para los niños de este rango de edad ( 7 a 9 años) lo que recomiendan como óptimo es de tamaño 16. Las características que desean y consideran importantes en los visualizadores son sonidos o alarmas para indicar eventos, como es el caso de advertir que se está cargando una nueva página, o colocar alguna animación para el desplazamiento de páginas, así como hacer más notorio el número que indica en que página están ubicados, también señalan que sería más ameno para ellos colocar animaciones en algunas escenas de la lectura.

\section{Historia}

El plan de estudio de la materia de Historia sigue un método inductivo para su enseñanza. En los dos primeros grados, el alumno adquiere y explota de manera elemental la noción del cambio a través del tiempo, utilizando como referencia las transformaciones que ha experimentado él y su familia, así como su entorno. Para los restantes grados el niño aprende los elementos más importantes de su entidad federativa, con un amplio componente narrativo. Para los niños en muchas ocasiones la Historia es difícil de comprender, la toman como una serie de datos que hay que memorizar y que posteriormente se aplica a un esquema de preguntas y respuestas, obteniéndose como resultado el olvido de los eventos. Una de las herramientas de enseñanza-aprendizaje que emplean los profesores son las líneas de tiempo, utilizadas al terminar el estudio de una época. Consiste en organizar a los niños en pequeños grupos para identificar etapas y períodos. Ellos encuentran como estructurar la historia de tal forma que se logre el cumplimento de la actividad trabajando colaborativamente e intercambiando conocimientos, logrando con ello la comprensión y noción del tiempo histórico. El sistema Edumóvil actualmente está llevando a cabo esta técnica por medio del uso de dispositivos móviles y siguiendo con la teoría de aprendizaje en forma colaborativa.

La aplicación colaborativa trabaja sobre dispositivos móviles utilizando el protocolo Bluetooth, propiciando el trabajo en equipo y el enriquecimiento de la interacción de los niños entre sus compañeros de clases, y fortaleciendo las relaciones sociales. La aplicación tiene contemplado trabajar por medio de un servidor que 
reparta líneas de tiempo a los diferentes equipos formados en clase, cada uno de ellos ordena los fragmentos de dicha línea trabajando en equipo, y posteriormente lo envía al servidor para ser evaluado (Figura 2).

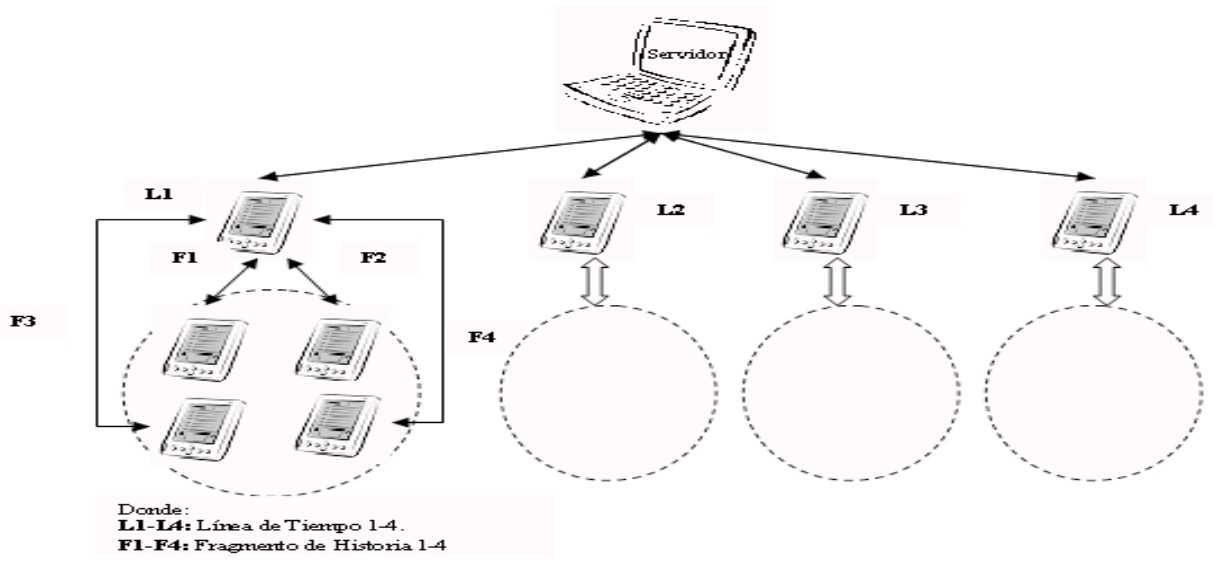

Figura 2

\section{Ciencias Naturales}

Los programas de Ciencias Naturales organizan su contenido en cinco ejes temáticos: 1) los seres vivos, 2) el cuerpo humano y la salud, 3) el ambiente y su protección, 4) materia, energía y cambio, y 5) ciencia, tecnología y sociedad. Edumóvil contempla que todos los puntos son importantes, pero actualmente se ha trabajado con los ejes de los seres vivos y, el ambiente y su protección, desarrollando un juego colaborativo llamado ¿Quién se come a quién? Dicho juego fue desarrollado para Palms con las siguientes características mínimas: 55 MB de RAM, pantalla de $320 x 480$ píxeles, Palm OS 5.4, procesador a $416 \mathrm{Mhz}$ y comunicación Bluetooth 1.o. Dicho juego puede ser utilizado en los grados de tercero a quinto como auxiliar en los temas donde el niño aprenda el comportamiento de un ecosistema y su crecimiento poblacional.

Las pruebas de usabilidad del juego se realizaron en una escuela primaria de la localidad, y en nuestro laboratorio. Lo que se observó en estas pruebas, fue que los niños si trabajaban en forma colaborativa, dado que charlaban para acordar como mantener el ecosistema en equilibrio, y que los dispositivos afectan el desempeño de los niños, dado que deben ser ligeros y pequeños, y deben ser del mismo modelo. En las pruebas realizadas se utilizaron las Palms Tungsten T5 y Lifedrive. 


\section{CONCLUSIONES}

Las pruebas de usabilidad han servido mucho de apoyo en Edumóvil, dado que han permitido identificar el tipo de PDA a utilizar, y las aplicaciones desarrolladas han sido probadas por los usuarios reales, de los cuales se ha recibido retroalimentación para su mejora. De dichas pruebas se ha notado que los dispositivos deben ser pequeños y ligeros, dado que puede afectar el desempeño del niño. Se deben realizar pruebas de usabilidad con los nuevos modelos de Palms para observar si son mejores que las elegidas actualmente, así como adaptar las aplicaciones desarrolladas a estos modelos.

Conociendo que los ejes de matemáticas donde los niños presentan dificultad de aprendizaje son los números, sus relaciones y sus operaciones para segundo, tercero y sexto grado de primaria, y proceso de cambio para quinto grado, debemos proponer aplicaciones para auxiliar en dichos ejes. Al contar con este conjunto de aplicaciones se podrá incorporar Edumóvil en una escuela primaria, y observar el impacto de la tecnología móvil en el proceso de enseñanza-aprendizaje.

El proyecto Edumóvil pretender ser una alternativa para auxiliar al profesor y al alumno en la forma de enseñanza-aprendizaje de ciertos temas de interés, así como en los temas en los cuales se detecten problemas en el aprendizaje tanto en forma individual como en forma colaborativa. No pretende sustituir al profesor, sino servir de apoyo en el salón de clases.

\section{REFERENCIAS BIBLIOGRÁFICAS}

Calzadilla, M. (2002). Aprendizaje colaborativo y tecnologías de la información y la comunicación. Revista Iberoamericana de Educación.

Druin, A.; Strommen, E.; Sacher, H.; Tatar, D. (2002). The word of wireless and Kids. CHI'O2. Minneapolis: ACM press, 704-705.

Gerónimo G.; Calvo I.; Rocha, E. (2005). Los Niños y los PDAs: una evaluación de su Uso. Puebla: Mexican Society of Computer Science.

Hanna, L.; Rinden, K. (1997). Guidelines for Usability Testing with Children. Interactions, 4 (5), 9-14.
Secretaría de Educación Pública. (2003). El plan y programas de estudio de educación básica primaria. México, D.F.: Fernández Editores.

Zurita, G.; Nussbaum, M.; Salinas, R. (2005). Dynamic Grouping in Collaborative Learning Supported by Wireless Handhelds. Educational Technology \& Society, 8 (3), 149-161.

Zurita, G.; Nussbaum, M. (2004). Computer supported collaborative learning using wirelesslyinterconnected handheld computers. Computer \& Education, 42, 289-314. 


\section{PERFIL ACADÉMICO Y PROFESIONAL DE LOS AUTORES}

Gabriel Gerónimo Castillo, Profesor-Investigador de la Universidad Tecnológica de la Mixteca. Responsable del Grupo de Investigación de Tecnología Aplicada a la Enseñanza. Ha participado en diferentes foros como VirtualEduca 2005, ENC 2005, VirtualEduca 2006, CLEI 2006, Interacción Persona-Ordenador 2006. Es Maestro en Ciencias de la Computación, y Licenciatura en Ciencias de la Computación.

E-mail: gcgero@nuyoo.utm.mx

Everth Haydeé Rocha Trejo, Profesora-Investigadora de la Universidad Tecnológica de la Mixteca. Ha participado en diferentes foros como VirtualEduca 2005, ENC 2005, VirtualEduca 2006, CLEI 2006. Es Maestra en Ciencias de la Computación, y Licenciatura en Ciencias de la Computación.

E-mail: everth@nuyoo.utm.mx

DIRECCIÓN DE LOS AUTORES

Universidad Tecnológica de la Mixteca,

Carretera a Acatlima km. 2.5,

Huajuapan de León, Oaxaca, México.

C.P. 69000.

Fecha de recepción de los artículos: 19/10/06

Fecha de aceptación de los artículos: 07/03/07 